\title{
Chemical Treatment Effects of Blue, Yellow and Green Colors with Heating Methods on Agates of Cheshme Shoor Area, Qom, Iran
}

\author{
Mohammad Yazdi' ${ }^{1}$, Reyhane Lotfi' ${ }^{1}$, Fariborz Masoudi' ${ }^{1}$, Niloofar Musavi Pak ${ }^{2}$ \\ ${ }^{1}$ Department of Geology, Shahid Beheshti University, Tehran, Iran \\ ${ }^{2}$ Incubation Center, Shahid Beheshti University, Tehran, Iran \\ Email: m_yazdi@sbu.ac.ir
}

Received 19 March 2016; accepted 9 May 2016; published 12 May 2016

Copyright (C) 2016 by authors and Scientific Research Publishing Inc.

This work is licensed under the Creative Commons Attribution International License (CC BY). http://creativecommons.org/licenses/by/4.0/

c) (i) Open Access

\section{Abstract}

The Cheshme Shoor area is located in northeast of Qom which is a part of central Iran geo-structural zone. The rocks of the area are mainly volcanic rocks that include Eocene acidic tuffs and ignimbrites. Different types of agate, jasper and amethyst formed as secondary cavity filling deposits in variety of colors and shapes in the area. In this paper, chemical treatments by different chemicals with heating methods in 15 different procedures have been conducted on one agate sample of the study area which has been divided into smaller pieces by milling. Dyeing with these methods has been done in blue, yellow and green colors. Excellent results have been achieved in 9 processes, significant but not expected results have been illustrated in 4 processes and 2 processes have been represented no changes. Produced colors have had a natural and light color which makes them more desirable to the consumers but totally these dyeing processes illustrate that concentration of the solution is effective in coloring and solutions with more concentrations produce stronger color.

\section{Keywords}

Treatment, Agate, Volcanic Rocks, Cheshme Shoor, Qom

\section{Introduction}

The study area is located in roughly $45 \mathrm{~km}$ northeast of Qom and near to Hoze Soltan Lake. The average altitude of the area is $1104 \mathrm{~m}$ above sea level. The volcanic rocks are dominant in the area which generally includes late Eocene acidic tuffs and ignimbrites but except these rocks, also Quaternary sedimentary formations are abun- 
dant in the area (Figure 1).

Gems are classified in two categories that include precious minerals (diamond, sapphire, ruby and emerald) and semi-precious minerals (agate, jade and other gems) [1]. According to the classification, gems of the study area have placed in semi-precious minerals group which contains amethyst, different types of agate with white to gray and a variety of jasper with brown, red, green and orange colors in volcanic rocks of the area.

Agate relates to silica rock from volcanic origin that is believed to form in the cavities created by vesiculation of volcanic host [2]. Agates have been observed in sedimentary, metamorphic and igneous environments and can be found in every continent. Agates forms as an infilling within some basaltic or rhyolithic gas vesicles but the mechanisms that produce agate in an igneous environment remain an enigma, also the silica sources, temperature, method of deposition, transportation and final mechanism of crystallization are all unknowns that have added to the difficulties in determining the origin of agate [3]. The majority of recent workers would accept that agates form at temperatures $<100^{\circ} \mathrm{C}$ [4] and consist of a variety of quartz. These minerals contain varying amount of water $\left(\mathrm{H}_{2} \mathrm{O}\right.$ and $\mathrm{Si}-\mathrm{OH}$ groups) which can be used to determine the mineral species present [5].

Some semi-precious minerals such as agate are not valuable because of their undesirable color but artificial dyeing can increase their economic value. The first records of treatments to change the color of gems are known from the Stockholm papyrus and the works by C. Pliny Secundus (23 - 76 AD). Some of these methods are used until today. Among the processes reported, the bleaching of crystals in boiling rice-water and the
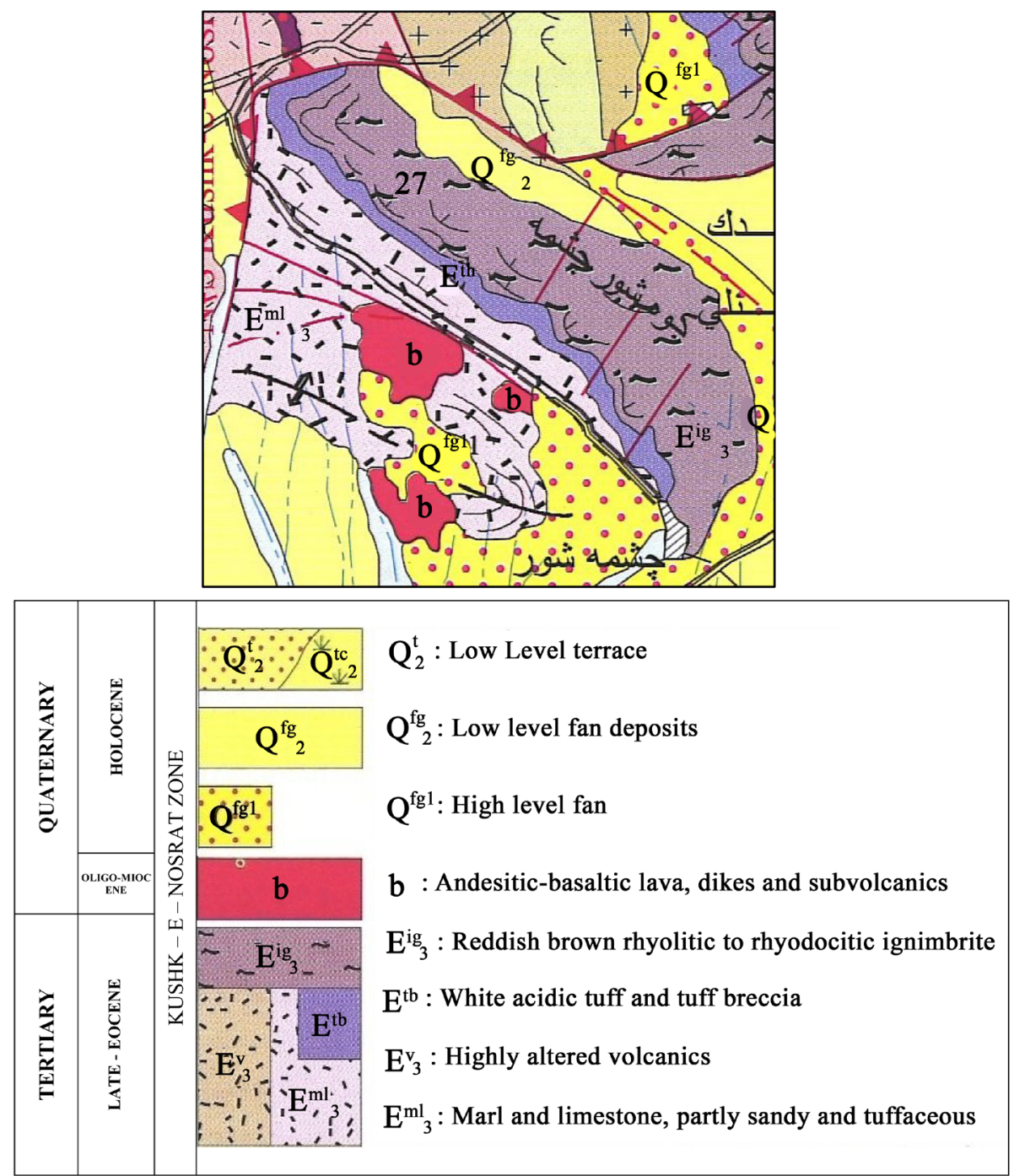

Figure 1. Geological map of the study area, derived from 1:100,000 geological map of Zaviyeh sheet. 
cleaning (bleaching) of smoky quartz in cauldrons of hot water at public baths are some of the first reports of thermal treatments of quartz done to improve their value. Even though the works of Pliny went through the Middle Ages as a reference in mineralogy and gemology, it was Albertus Magnus who realized for the first time that the color of gemstones was associated to their composition [6].

Three main methods that are used for dyeing semi-precious minerals involve heating, using chemicals and radioactive radiation. Using heat to improve gems color is a very old method (as mentioned above) [6]. Using chemicals or dyeing is also one of the oldest methods to create colors in gemstones [6], which is used for agates in this article. Color absorption varies in different types of agates and depends on the porosity and water content of each layer. Brighter layers composed of dense quartz crystals so do not absorb color well or absorb a little color. Layers that absorb color easier are called soft layers and layers that do not absorb colors well are known as hard layers [7].

With the advent of radioactivity, discovered at the end of the nineteenth and at the beginning of the twentieth century, its application to change the gemstone colors was almost immediate and one of the most effective ways to increase the value of pale or colorless quartz is combining irradiation with thermal treatment. After faceting, gamma irradiation and/or thermal treatment is used to produce the colored quartz varieties in order to increase the value of the primary colorless quartz [6].

This paper is organized as follows. In Section 2, we investigate several methods of dyeing which include blue, yellow and green colors by different chemicals and solutions with or without thermal treatments. Figures have shown used chemicals, results and before/after pictures of processes. Finally we give a brief conclusion in the last section.

\section{Chemical Treatments and Effects on Agates of the Study Area}

In this research, chemical treatments with heating methods based on George W. Fischer [8] have been used to enhance color of agates. Gemological investigations on a number of semi-precious minerals especially finely-cut agates have revealed that they probably have had coloration capabilities. Furthermore, thermal improvements on agates and a jasper of this area have been done by Lotfi et al. [9]. For chemical treatments a single tube agate has used which had both chalcedony and crystalline parts. The agate sample has been divided into smaller pieces by milling then they have been washed by water, finally they have dried for 20 hours at $37^{\circ} \mathrm{C}$ by electric heater (Figure 2). Due to the high solubility of some of the chemicals, at the beginning of the processes, chemicals

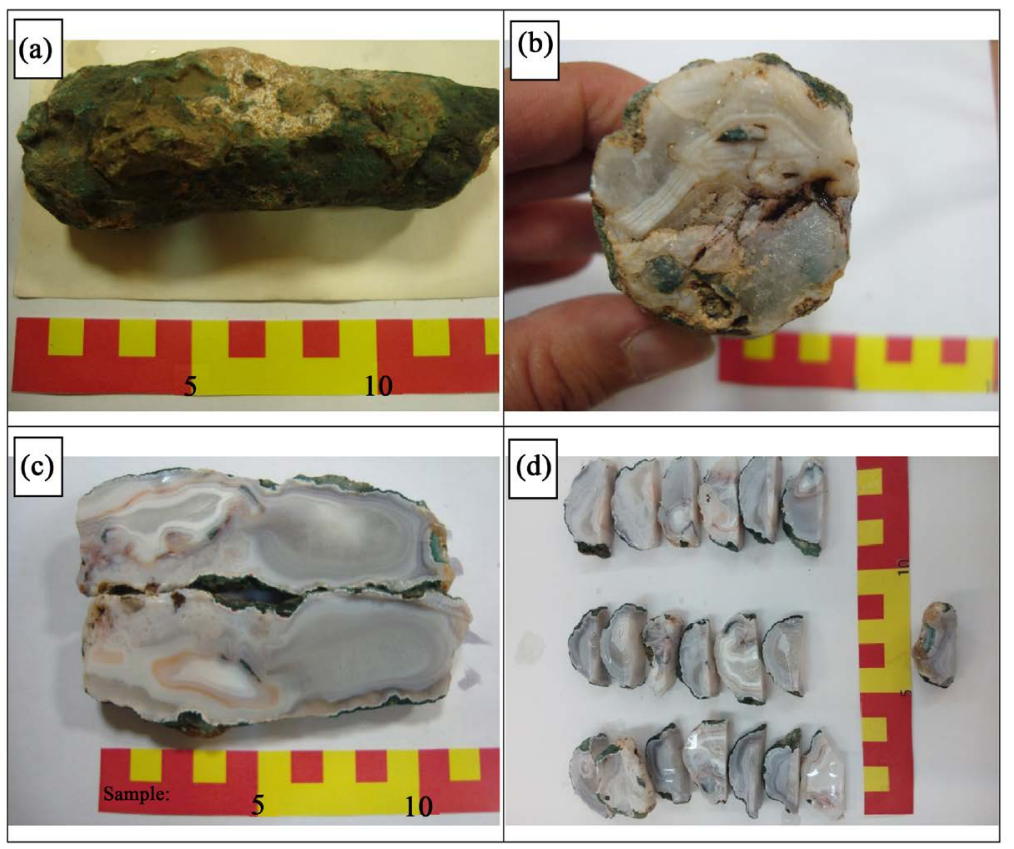

Figure 2. (a) Sample’s length; (b) sample's width; (c) sample's length cut into two parts; (d) sliced and washed samples before drying. 
have poured into the containers then water slowly have been added to substances. After bringing agates samples out of the solution, they have been washed and dried. To satisfy more accurate of depth and intensity of color's penetration in processes, all samples have been polished by gem cutting machine after the completion of dyeing.

\subsection{Kinds of Blue}

To evaluate the effect of blue on agates, different kinds of chemicals have been used in different processes. These enhancement results have been shown in Figure 3. Except first process (copper nitrate), rest of processes have had two phases.

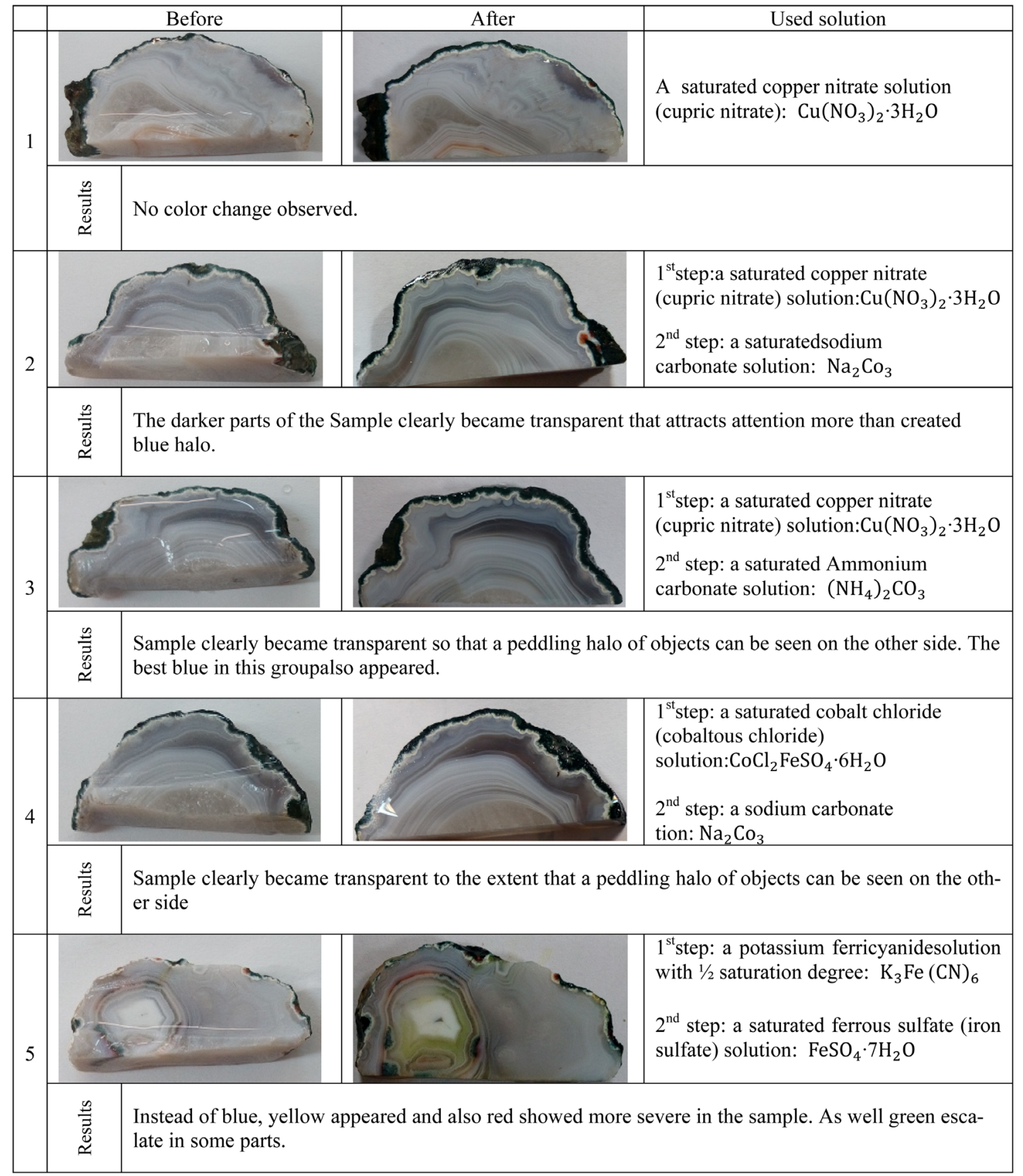

Figure 3. The effect of blue dyeing processes on samples with used materials and observed changes. 


\subsubsection{Copper Nitrate}

This is a simple process, single-stage and single solution. The process produces only rather light shades of blue even from a saturated solution and heating the sample in an oven at the lowest temperature for several hours probably could obtain a stronger color [8]. It has showed the weakest results in blue group.

\subsubsection{Copper Nitrate-Sodium Carbonate}

The results of this process should be a bit stronger than copper nitrate solution alone. Blending water and sodium carbonate in beaker is an exothermal reaction that makes heat noticeably so intervention, adding water or extra response is not required.

\subsubsection{Copper Nitrate-Ammonium Carbonate}

This process should make deeper blue than previous processes [8], but in the study area's agate sample, increasing the transparency of the sample was more significant than the created blue color and generally this process has had the best results among the blue color processes. Mixing ammonium carbonate with water is an endothermal reaction which makes container cold. During 24 hours of making solution, foam will produced and contaminate periphery.

\subsubsection{Cobalt Chloride-Sodium Carbonate}

This process needs a heating step. The reaction between sodium carbonate and water is exothermic and makes container warmer noticeably so there is no need to intervention and adding water or extra responses.

\subsubsection{Potassium Ferricyanide-Ferrous Sulfate}

This process requires a heating step.

\subsection{Kinds of Yellow}

To evaluate the effects of different processes of yellow color enhancements on agates, some kinds of chemicals have been used in different processes. Yellow color have had the best effects and significant results on the agate samples overall. Enhancement results of yellow color have been presented in Figure 4.

\subsubsection{Chromium Trioxide}

In this process, a strong chromium trioxide solution is required. After removing the sample out of the solution, it has been soaked in pure water for three days until additional chemical substance gets out. Chromium trioxide is very soluble, so water should be added gradually to it. By changing the density of the solution, it is possible to obtain a range of yellow color.

\subsubsection{Nickel Nitrate-Sodium Dichromate}

Before dissolving all nickel nitrate, should stop adding water to the beaker. As it is obvious in Figure 4, this process has showed the strongest yellow in the group. This process requires a heating step as well.

\subsubsection{Sodium Dichromate}

This procedure has had the weakest result in yellow group. Other dichromates can be used for this process but sodium dichromate is the cheapest dichromate and is very simple to use. For example, staining with ammonium dichromate is attractive as sodium dichromate but it is more expensive than sodium dichromate or potassium dichromate can also make the color less intense [8].

\subsubsection{Iron Chloride}

This is a simple process, single-stage and single solution and finally could produce a brown-yellow color [8]. Adding chemicals should be continued until all of the crystals remain dissolved in the solution.

\subsubsection{Potassium Permanganate}

This is another simple and single step method that could make a brown to brown-purple color especially on translucent gems [8]. For clean the excess potassium permanganate of sample, after bring the sample out of solution, it has been soaked in pure water for three days and meanwhile the water was changed several times. 


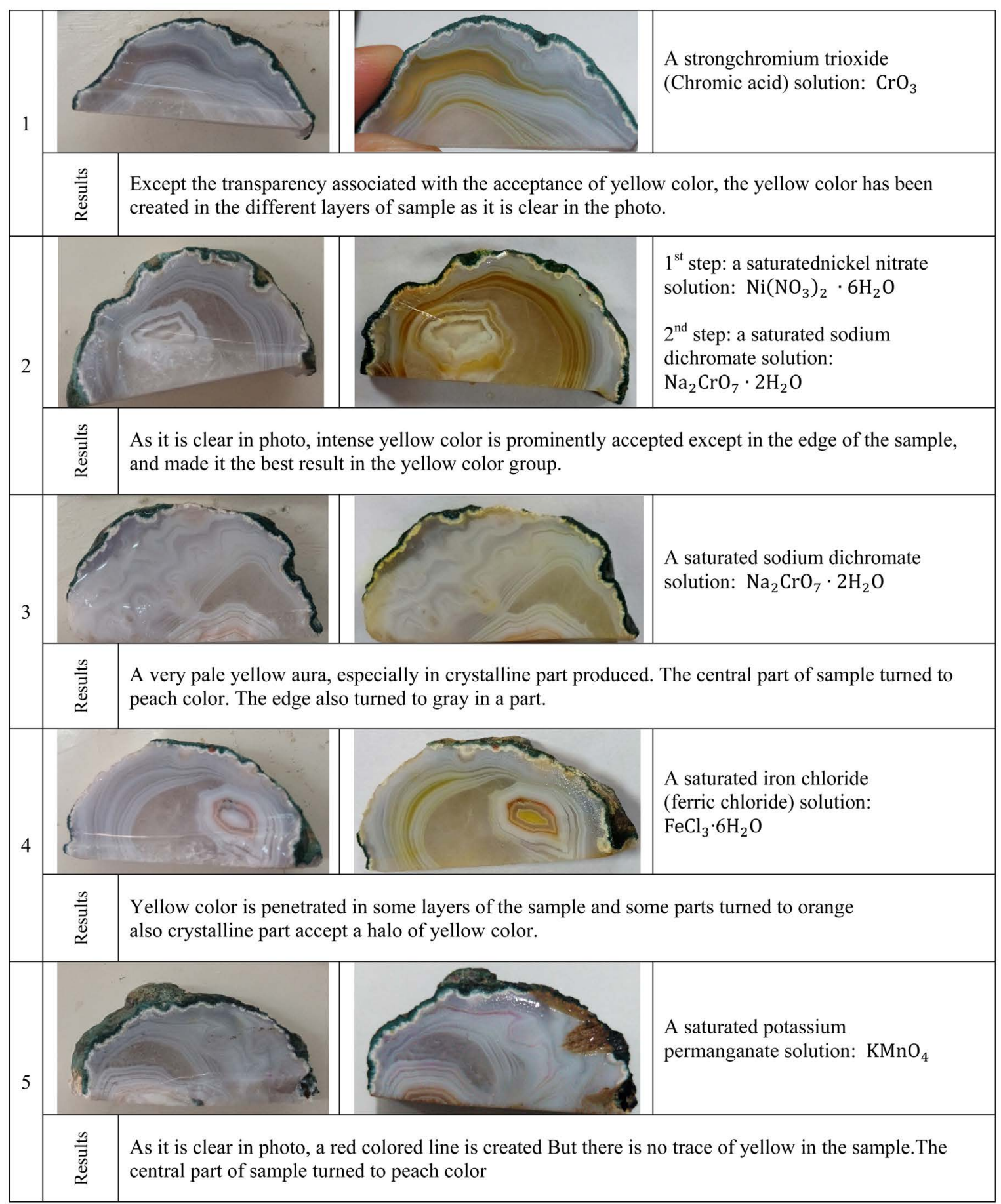

Figure 4. The effect of yellow dyeing processes on samples with used materials and observed changes.

\subsection{Kinds of Green}

This group has had the hardest procedures and the weakest results. Enhancement results of green color, has shown in Figure 5.

\subsubsection{Chromium Chloride}

Chromium chloride should provide the best color in the green color group [8] but it was one of the weakest. 


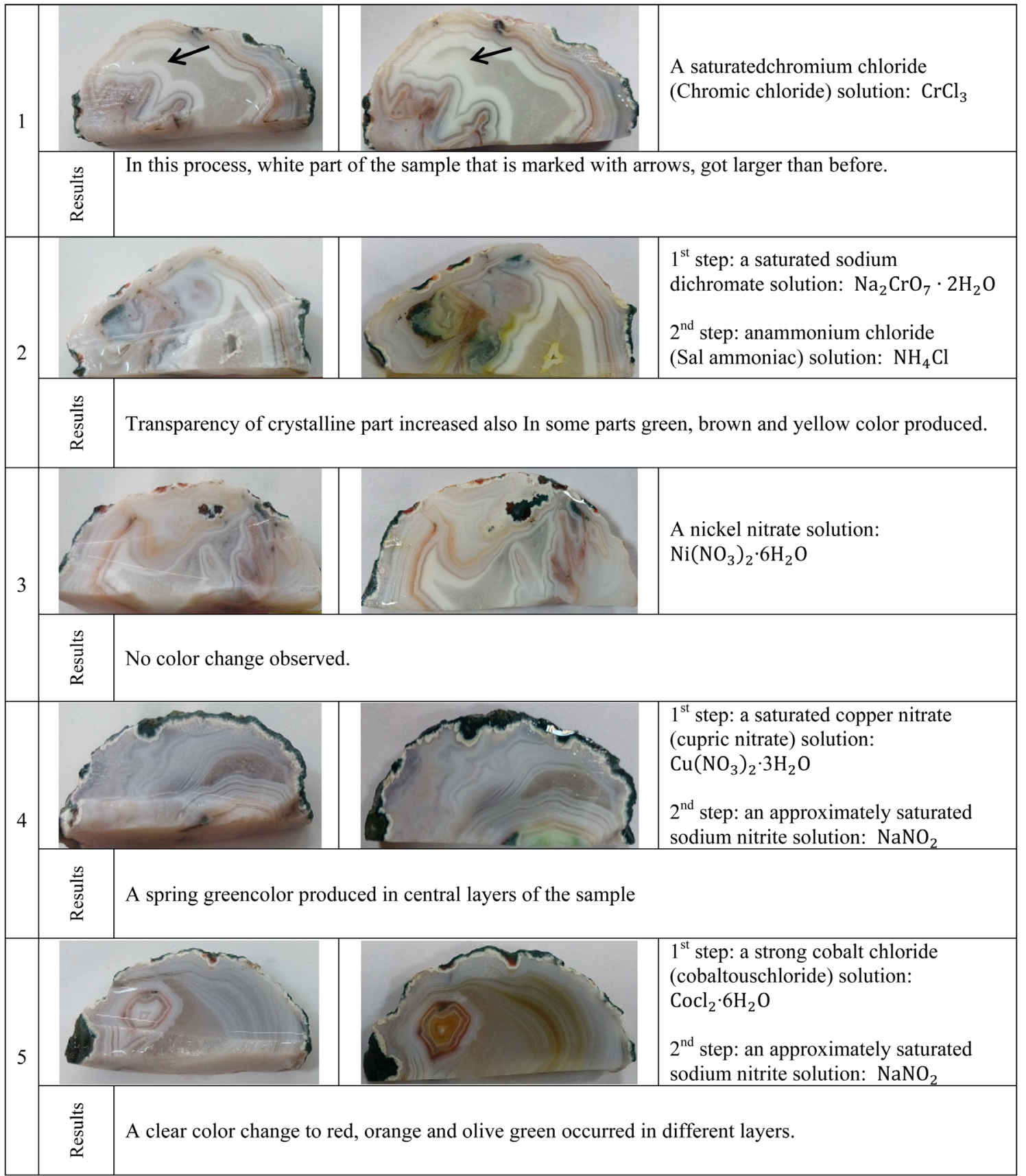

Figure 5. The effect of green dyeing processes on samples with used materials and observed changes.

\subsubsection{Sodium Dichromate-Ammonium Chloride}

After completing the first step, for preparing the final solution, ammonium chloride should be mixed with sodium dichromate solution. After that, sample has been placed in the mixture. In fact added ammonium chloride to sodium dichromate solution is a promoter in the decomposition of sodium dichromate and the process does not complete without it. Making ammonium chloride solution is endothermal so it is natural that container get cooled. This process requires two heating steps. In this process the heat of baking step breaks down sodium dichromate to chromium oxide $\left(\mathrm{Cr}_{2} \mathrm{O}_{3}\right)$, which is a strong and insoluble green pigment in the painting industry that known as chrome green. Since the pigment is insoluble even in the non-aqueous solvents, there is no other way to saturate the gem with this substance except this procedure [8]. 


\subsubsection{Nickel Nitrate}

This is a simple and single step process. Nickel nitrate is very soluble and should be added until quantitative of insoluble salt left in the bottom of the container.

\subsubsection{Copper Nitrate-Sodium Nitrite}

The second phase solution (sodium nitrite) is an endothermal reaction so containers cooling is completely natural.

\subsubsection{Cobalt Chloride-Sodium Nitrite}

The quantity of cobalt chloride could be more or less. The cobalt chloride solution can also be used for other processes. This process requires a heating step.

\section{Conclusion}

Chemical treatment with heating is the most possible method of dyeing for Cheshme Shoor area's agates. Produced colors have had light and smooth tones which make them more desirable to the consumers. After finishing the processes, the chalcedony parts have showed the most color change while the crystals at the best conditions have presented a halo of color. Generally this study has been conducted based on 15 different processes in three colors (blue, yellow and green) and indicates excellent dyeing results in 9 processes, tangible changes but not expected changes in 4 processes and finally 2 samples remain unchanged. Excellent results contain samples 2, 3 and 4 in blue group, 1, 2 and 4 of yellow group and 2, 4 and 5 in green color group, 4 unexpected results include process 5 in blue group, 3 and 5 of yellow group and number 1 in green color group and eventually number 1 in blue group and 3 in green color group remain unchanged. Yellow color group has had the best results of all. Another major finding of these treatments is that the concentration of the solution is effective in coloring and solutions with more concentrations produce stronger color.

\section{References}

[1] Yazdi, M. (2008) Crystallography. Ball Press, Tehran.

[2] Moxon, T., Nelson, D.R. and Zhang, M. (2006) Agate Recrystallisation: Evidence from Samples Found in Archaean and Proterozoic Host Rock, Western Australia. Australian Journal of Earth Sciences, 53, 235-248. http://dx.doi.org/10.1080/08120090500499255

[3] Moxon, T. (2002) Agate: A Study of Ageing. European Journal of Mineralogy, 14, 1109-1118. http://dx.doi.org/10.1127/0935-1221/2002/0014-1109

[4] Moxon, T. and Reed, S.J.B. (2006) Agate and Chalcedony from Igneous and Sedimentary Hosts Aged from 13 to 3480 Ma: A Cathodoluminescence Study. Mineralogical Magazine, 70, 485-498. http://dx.doi.org/10.1180/0026461067050347

[5] Graetsch, H., Flörke, O.W. and Miehe, G. (1985) The Nature of Water in Chalcedony and Opal-C from Brazilian Agate Geodes. Physics and Chemistry of Minerals, 12, 300-306. http://dx.doi.org/10.1007/BF00310343

[6] Götze, J. and Möckle, R. (2012) Quartz: Deposits, Mineralogy and Analytics. Springer, Berlin. http://dx.doi.org/10.1007/978-3-642-22161-3

[7] Sheikhi Gheshlaghi, R. (2014) Volcanism of South-West Troud and Its Relationship with Formation of Semi-Precious Rocks. M.Sc. Thesis, Shahid Beheshti University, Tehran.

[8] Fischer, G.W. (1990) Gemstone Coloration and Dyeing. http://www.ganoksin.com/borisat/nenam/index-gemstone-dyeing

[9] Lotfi, R., Yazdi, M. Masudi, F. and Musavi Pak, N. (2015) Gemological Characteristics and Thermal Treatment Effects on Semi-Precious Minerals in the Cheshme Shoor, Qom. 33rd National Geosciences Symposium, Tehran, 22-23 February, 1-7. 